PROCEEDINGS OF THE

AMERICAN MATHEMATICAL SOCIETY

Volume 133, Number 11, Pages 3281-3284

S 0002-9939(05)08035-4

Article electronically published on May 2, 2005

\title{
ALGEBRAS OF OPERATORS AND CLOSED RANGE
}

\author{
WARREN R. WOGEN
}

(Communicated by David R. Larson)

\begin{abstract}
We consider operators $T$ such that every operator in the norm closed algebra generated by $T$ has closed range. Examples in a triangular AF algebra are constructed.
\end{abstract}

\section{INTRODUCTION}

In this note we consider closed range properties for certain families of Hilbert space operators. Our motivation is the following question raised by Zemanek. (See Z p. 379].)

Question 1. If $T$ is a quasinilpotent operator and if $T^{n}$ has closed range for all $n \in \mathbb{N}$, must $T$ be nilpotent?

This question arose from results of Mbekhta and Zemanek, [MZ, Theorem 2], concerning uniform convergence of operator ergodic averages. An equivalent question is

Question 2. If $\sigma(T)$ is finite and if $p(T)$ has closed range for every polynomial $p$, must $T$ be algebraic?

L. Burlando and the author independently produced examples (unpublished) that answer these questions in the negative.1 However, it turns out that these questions had been answered in a strong way by Apostol in [A1]. (See also [A2].) Suppose that $\mathcal{H}$ is a complex Hilbert space and that $\mathcal{B}(\mathcal{H})$ denotes the algebra of bounded linear operators on $\mathcal{H}$. Apostol proved these theorems in [A1].

Theorem 3. If $T \in \mathcal{B}(\mathcal{H})$ and if $T-\lambda$ has closed range for all $\lambda \in \mathbb{C}$, then $\sigma(T)$ is countable.

Theorem 4. If $\sigma$ is a nonempty countable compact subset of $\mathbb{C}$, then there is an operator $T \in \mathcal{B}(\mathcal{H})$ (here $\operatorname{dim} \mathcal{H}$ is infinite) such that $\sigma(T)=\sigma$ and so that $p(T)$ has closed range for every polynomial $p$.

Apostol's construction is subtle and intricate. However, for the case $\sigma=\{0\}$, one can check that the operator produced is not nilpotent.

In this note we prove analogs of Theorems 3 and 4 . Given $T \in \mathcal{B}(\mathcal{H})$, let $\mathcal{A}(T)$ be the norm closed algebra generated by $T$. Thus $\mathcal{A}(T)$ is the norm closure of the

Received by the editors June 11, 2004.

2000 Mathematics Subject Classification. Primary 47A05; Secondary 47C05, 47L40.

${ }^{1}$ Added in proof: Burlando's example will appear in a paper, "On nilpotent operators", to appear in Studia Math. 
polynomials in $T$. Clearly $\mathcal{A}(T)$ is finite dimensional precisely when $T$ is algebraic. We will prove these theorems.

Theorem 5. If $T \in \mathcal{B}(\mathcal{H})$ and if $S$ has closed range for all $S \in \mathcal{A}(T)$, then $\sigma(T)$ is finite.

Theorem 6. If $\sigma$ is a nonempty finite subset of $\mathbb{C}$, then there is a nonalgebraic operator $T \in \mathcal{B}(\mathcal{H})$ with $\sigma(T)=\sigma$ and so that $S$ has closed range for all $S \in \mathcal{A}(T)$.

For the case that $\sigma=\{0\}$ in Theorem 6 , we produce a countably generated noncommutative subalgebra $\mathcal{A}^{0}$ of a familiar triangular AF algebra such that $S$ has closed range for all $S \in \mathcal{A}^{0}$.

The author wishes to thank J. Peters for helpful discussions on this work.

\section{RESULTS}

We begin by proving Theorem 5 . If $T$ satisfies the hypotheses of that theorem, then $\sigma(T)$ is countable, by Theorem 3. If $\sigma(T)$ is also infinite, then we will construct $S \in \mathcal{A}(T)$ so that the range of $S$ is not closed.

Every countable compact set has isolated points, so we can choose a sequence $\left(\lambda_{n}\right)$ such that each $\lambda_{n}$ is isolated in $\sigma(T)$. For each $n$, let $P_{n}$ be the Riesz idempotent corresponding to $\left\{\lambda_{n}\right\}$ determined by the Riesz Functional Calculus (see C. p. 210]). Then $P_{n} \in \mathcal{A}(T), 0 \neq P_{n}=P_{n}^{2}$, and $P_{n} P_{m}=0$ if $m \neq n$.

Let $S=\sum_{n=1}^{\infty} \frac{P_{n}}{4^{n}\left\|P_{n}\right\|}$. Then $S \in \mathcal{A}(T)$. For all $n$, let $x_{n}$ be a unit vector in $P_{n} \mathcal{H}$. Then

$$
S\left(\sum_{n=1}^{N} 2^{n}\left\|P_{n}\right\| x_{n}\right)=\sum_{n=1}^{N} \frac{1}{2^{n}} x_{n}
$$

so $\sum_{n=1}^{\infty} \frac{1}{2^{n}} x_{n}$ is in the closure of the range of $S$. But if $y \in \mathcal{H}$ and if $S y=$ $\sum_{n=1}^{\infty} \frac{1}{2^{n}} x_{n}$, then

so that

$$
\frac{1}{2^{n}} x_{n}=P_{n} S y=\frac{P_{n} y}{4^{n}\left\|P_{n}\right\|},
$$

$$
\left\|P_{n} y\right\| \geq 2^{n}\left\|P_{n}\right\| \quad \text { and } \quad\|y\| \geq 2^{n} .
$$

This holds for all $n$, so we have a contradiction.

We proceed to the proof of Theorem 6 . It will suffice to find a nonnilpotent quasinilpotent operator $A$ such that $S$ has closed range for all $S \in \mathcal{A}(A)$. For then, given $\sigma=\left\{\lambda_{1}, \lambda_{2}, \cdots, \lambda_{N}\right\} \subset \mathbb{C}$, the operator $T=\bigoplus_{n=1}^{N}\left(A-\lambda_{n} I\right)$ has the required properties. As noted in the introduction, we show that $A$ can be chosen to be any nonnilpotent operator in a certain ideal $\mathcal{A}^{0}$ of the triangular $\mathrm{AF}$ algebra known as the refinement algebra.

We outline the construction of $\mathcal{A}^{0}$. ( $\mathcal{A}^{0}$ was also considered in [PW] Remark 2.18] in another setting.) Let $M_{k}$ denote the $k$ by $k$ complex matrices. For all $n \in \mathbb{N}$, $\left\{e_{i, j}^{(n)}: 1 \leq i . j \leq 2^{n}\right\}$ denotes the matrix units in $M_{2^{n}}$, and $\nu_{n}: M_{2^{n}} \rightarrow M_{2^{n+1}}$ denotes the refinement embedding given by

$$
\nu_{n}\left(e_{i, j}^{(n)}\right)=e_{2 i-1,2 j-1}^{(n+1)}+e_{2 i, 2 j}^{(n+1)} .
$$

Let $\mathcal{T}_{n}$ be the upper triangular subalgebra of $M_{2^{n}}$ and let $\mathcal{T}_{n}^{0}$ be the strictly upper triangular subalgebra. The inductive $\operatorname{limit} \mathcal{T}=\lim \left(\mathcal{T}_{n}, \nu_{n}\right)$ is called the refinement algebra. $\mathcal{T}$ is a norm closed subalgebra of the UHF $2^{\infty} C^{*}$-algebra, or CAR algebra. (See $[\mathrm{KR}$, p. 759 , for example.) In what follows, we can consider $\mathcal{T}$ as faithfully 
represented as a subalgebra of $\mathcal{B}(\mathcal{H})$ for some $\mathcal{H}$. In [D], Donsig showed that $\mathcal{T}^{0}=$ $\lim \left(\mathcal{T}_{n}^{0}, \nu_{n}\right)$ is the Jacobsen radical of $\mathcal{T}$. Thus $\sigma(T)=\{0\}$ if $T \in \mathcal{T}^{0}$.

For $n \in \mathbb{N}$, let $X_{n}=e_{2^{n}-1,2^{n}}^{(n)}$ and suppose that $\mathcal{A}_{0}$ is the norm closed algebra generated by $\left\{X_{n}\right\}_{n=1}^{\infty}$. Note that $\mathcal{A}^{0} \subset \mathcal{T}^{0}$. Let $\mathcal{A}=\mathbb{C} I+\mathcal{A}^{0}$.

We analyze the structure of $\mathcal{A}$ in more detail. First we consider the matrix units in $\mathcal{A}$. Observe that $X_{m} X_{n}=0$ if $m \geq n$. If $m<n$, then $X_{m} X_{n}$ is a matrix unit in $M_{2^{n}}$.

For $n \in \mathbb{N}$, let $S_{n}$ be the set of all ordered subsets $K$ of $\mathbb{N}$ such that $n=\max K$. So if $K \in S_{n}$, then $K=\{n\}$ or $K$ has the form $\left\{k_{1}<k_{2}<\cdots<k_{\ell}<n\right\}$. In the latter case, let $X_{K}$ denote $X_{k_{1}} X_{k_{2}} \cdots X_{k_{\ell}} X_{n}$, and let $X_{\{n\}}=X_{n}$. Also let $X_{\emptyset}=I$. Define $\theta: S_{n} \rightarrow \mathbb{N}$ by $\theta(\{n\})=2^{n}-1$, and $\theta(K)=2^{n}-\left(\sum_{j=1}^{\ell} 2^{n-k}\right)-1$ if $K=\left\{k_{1}<k_{2}<\cdots<k_{\ell}<n\right\}$. A computation shows the following.

(7) If $K \in S_{m}$, then $X_{K}=e_{\theta(K), 2^{n}}^{(n)}$. Thus $K \mapsto X_{K}$ sets up a 1-1 correspondence from $S_{m}$ onto $\left\{e_{i, 2^{n}}^{(n)}: 1 \leq i<2^{n}, i\right.$ odd $\}$.

It is now easy to check that if $K_{1}$ and $K_{2}$ are distinct finite subsets of $\mathbb{N}$, then $X_{K_{1}}$ and $X_{K_{2}}$ are disjoint in the following sense: For any $n$ large enough that both $X_{K_{1}}$ and $X_{K_{2}}$ are in $M_{2^{n}}$, the matrix entry of $X_{K_{1}}$ in $M_{2^{n}}$ is 0 at each position for which the matrix entry of $X_{K_{2}}$ is 1 . It follows easily that each element of $\mathcal{A}$ is a series $\sum_{K} a_{K} X_{K}$, where $K$ ranges over the finite subsets of $\mathbb{N}$. We show in Remark 12 that the coefficients $\left(a_{K}\right)$ must be in $\ell^{2}$.

Next we show that $\mathcal{A}$ has a fractal-like behavior; $\mathcal{A}$ replicates itself relative to each matrix unit $X_{J}$. To make this precise, fix $X_{J}$ with $J \in S_{n}$. Then $d\left(X_{J}\right)=$ $X_{J}^{*} X_{J}=e_{2^{n}, 2^{n}}^{(n)}$ is the domain of $X_{J}$ and $r\left(X_{J}\right)=X_{J} X_{J}^{*}$ is the range of $X_{J}$. Denote the compression of $\mathcal{A}$ to $X_{J}$ by $\mathcal{A}_{J}=r\left(X_{J}\right) \mathcal{A} d\left(X_{J}\right)$. One can check that $X_{K} \in \mathcal{A}_{J} \Leftrightarrow J$ is the initial segment of $K$. (That is, either $K=J$ or $K=J \cup K^{\prime}$ for some $K^{\prime}$ with $m$ in $K^{\prime}>n$.)

Finally, observe that the matrix units in $X_{J}^{*} \mathcal{A}_{J}$ have domain and range inside $D\left(X_{J}\right)$.

(8) $\mathcal{A}$ and $X_{J}^{*} \mathcal{A}_{J \mid d\left(X_{J}\right)}$ are isometrically isomorphic as unital operator algebras.

In fact, define $\psi: \mathcal{A} \rightarrow X_{J}^{*} \mathcal{A}_{J}$ by $\psi\left(\sum a_{K} X_{K}\right)=X_{J}^{*} \sum a_{K} X_{J} X_{K+\{n\}}$. Check that the matrix pictures for $A \in \mathcal{A}$ and $\psi(A)_{\mid d\left(X_{J}\right)}$ are identical.

Theorem 9. Every $A \in \mathcal{A}$ has closed range.

Proof. Note that if $A \in \mathcal{A}$, then $A=a_{\emptyset} I+A^{0}$ where $A^{0} \in \mathcal{A}^{0}$. So if $a_{\emptyset} \neq 0$, then $A$ is invertible. Thus it suffices to assume that $A \in \mathcal{A}^{0}$. Then $A=\sum a_{K} X_{K}$, where $K$ ranges over the nonempty finite subsets of $\mathbb{N}$. We will need the following observation about $n \times n$ operator matrices with only one nontrivial column.

Lemma 10. Suppose that $T=\left(T_{i, j}\right)_{i, j=1}^{n} \in \mathcal{B}\left(\bigoplus_{i=1}^{n} \mathcal{H}\right)$. Suppose that $T_{i, j}=0$ unless $j=n$ and that $T_{i_{0}, n}$ is invertible for some $i_{0}$. Then $T$ has closed range.

Proof. $\operatorname{ker} T$ is the direct sum of the first $n-1$ copies of $\mathcal{H}$. Since $T_{i_{0}, n}$ is invertible, $T$ is bounded below on $(\operatorname{ker} T)^{\perp}$, so $T$ has closed range.

We apply this lemma to $A=\sum a_{K} X_{K} \in \mathcal{A}^{0}$. Choose the smallest $n$ such that $a_{J} \neq 0$ for some $J \in S_{n}$. Then $A$ has a $2^{n}$ by $2^{n}$ block matrix as in Lemma 10 , and one block, $A_{J}=r\left(X_{J}\right) A d\left(X_{J}\right)$, is invertible. In fact, referring to (8), we have that 
$\psi^{-1}\left(X_{J}^{*} A_{J}\right)=a_{J} I+B_{0}$ for some $B^{0} \in \mathcal{A}^{0} . a_{J} \neq 0$, so $A_{J}$ is invertible. Lemma 10 shows that $A$ has closed range.

\section{CONCLUDing REMARKS}

Remark 11. To finish the proof of Theorem 6, it suffices to choose a nonnilpotent operator $T$ in $\mathcal{A}^{0}$. For example, $T=\sum \frac{1}{2^{n}} X_{n}$ does the job.

Remark 12. There are many ways to represent the refinement algebra $\mathcal{T}$ as an algebra of operators. For example, let $\mathcal{H}=\ell^{2}(B)$ where $B$ denotes the dyadic rationals in $(0,1] .\left\{f_{r}: r \in B\right\}$ is a basis for $\mathcal{H}$, where $f_{r}(s)=\delta_{r s}, s \in B$. If $e_{i j}^{(n)} \in M_{2^{n}}$, let

$$
e_{i j}^{(n)} f_{r}= \begin{cases}f_{r+\frac{i-j}{2^{n}}}, & r \in\left(\frac{j-1}{2^{n}}, \frac{j}{2^{n}}\right], \\ 0, & \text { otherwise. }\end{cases}
$$

Using (7), we see that the map $K \mapsto X_{K} f_{1}$ is $1-1$ from $S_{n}$ onto $\left\{f_{\frac{i}{2^{n}}}: 1 \leq i<\right.$ $2^{n}, i$ odd $\}$. This holds for all $n \in \mathbb{N}$, so the map $X_{K} \rightarrow X_{K} f_{1}$ carries the matrix units in $\mathcal{A}$ onto a basis for $\mathcal{H}$. Thus $f_{1}$ is a cyclic vector for $\mathcal{A}$. In addition, if $A=\sum a_{K} X_{K} \in \mathcal{A}$, then $\left\|A f_{1}\right\|^{2}=\sum\left|a_{K}\right|^{2}$, so $f_{1}$ is a separating vector, and

$$
\left\|\sum a_{K} X_{K}\right\|^{2} \geq \sum\left|a_{K}\right|^{2}
$$

Denote the right-hand side of this inequality as the $\ell^{2}$ norm of $A$.

It is unknown whether the operator norm and the $\ell^{2}$ norm are equivalent on $\mathcal{A}$. The two norms agree on $A=\sum a_{K} X_{K}$ provided those $X_{K}$ with $a_{K} \neq 0$ have pairwise orthogonal ranges. The equivalence of these norms would imply that every representation of $\mathcal{A}$ is closed in the weak operator topology.

\section{REFERENCES}

[A1] C. Apostol, On the closed range points in the spectrum of operators, Rev. Roum. Math. Pures et Appl 21 (1976), 971-975. MR0425639 (54:13593)

[A2] C. Apostol, The reduced minimum modulus, Michigan Math. J. 32 (1985), 279-294. MR0803833 (87a:47003)

[C] J.B. Conway, A Course in Functional Analysis, 2nd Ed., Springer-Verlag, New York, 1990. MR.1070713 (91e:46001)

[D] A. Donsig, Semisimple triangular AF algebras, J. Funct. Anal. 111 (1993), 323-349. MR 1203457 (94b:46084)

[KR] R.V. Kadison and J.R. Ringrose, Fundamentals of the Theory of Operator Algebras, Vol. II, Academic Press, Orlando, 1986. MR0859186 (88d:46106)

[MZ] M. Mbekhta and J. Zemanek, Sur le théorème ergodique uniform et le spectra, C.R. Acad. Sci. Paris Ser. I. Math. 317 (1993), 1155-1158. MR.1257230 (95b:47010)

[PW] J.R. Peters and W.R. Wogen, Commutative radical operator algebras, J. Operator Theory 42 (1999), 405-424. MR1717020 (2000j:47124)

[Z] J. Zemanek, On the Gelfand-Hille theorems, in Functional Analysis and Operator Theory, Banach Center Publication 30, Polish Academy of Sciences, Warszawa, 1994, 369-385. MR:1285622 (95f:47009)

Department of Mathematics, University of North Carolina, Chapel Hill, North CarOLINA 27599-3250

E-mail address: wrw@email.unc.edu 